\title{
THE INFIMUM IN THE METRIC MAHLER MEASURE
}

\author{
CHARLES L. SAMUELS
}

\begin{abstract}
Dubickas and Smyth defined the metric Mahler measure on the multiplicative group of non-zero algebraic numbers. The definition involves taking an infimum over representations of an algebraic number $\alpha$ by other algebraic numbers. We verify their conjecture that the infimum in its definition is always achieved as well as establish its analog for the ultrametric Mahler measure.
\end{abstract}

\section{INTRODUCTION}

Let $K$ be a number field and $v$ a place of $K$ dividing the place $p$ of $\mathbb{Q}$. Let $K_{v}$ and $\mathbb{Q}_{p}$ denote the respective completions. We write $\|\cdot\|_{v}$ for the unique absolute value on $K_{v}$ extending the $p$-adic absolute value on $\mathbb{Q}_{p}$ and define

$$
|\alpha|_{v}=\|\alpha\|_{v}^{\left[K_{v}: \mathbb{Q}_{p}\right] /[K: \mathbb{Q}]}
$$

for all $\alpha \in K$. Define the Weil height of $\alpha \in K$ by

$$
H(\alpha)=\prod_{v} \max \left\{1,|\alpha|_{v}\right\}
$$

where the product is taken over all places $v$ of $K$. Given this normalization of our absolute values, the above definition does not depend on $K$, and therefore, $H$ is a well-defined function on $\overline{\mathbb{Q}}$. Clearly $H(\alpha) \geq 1$, and by Kronecker's Theorem, we have equality precisely when $\alpha$ is zero or a root of unity. It is obvious that if $\zeta$ is a root of unity then

$$
H(\alpha)=H(\zeta \alpha),
$$

and further, if $n$ is an integer then it is well-known that

$$
H\left(\alpha^{n}\right)=H(\alpha)^{|n|} .
$$

Also, if $\alpha, \beta \in \overline{\mathbb{Q}}^{\times}$then $H(\alpha \beta) \leq H(\alpha) H(\beta)$ so that $H$ satisfies the multiplicative triangle inequality.

We further define the Mahler measure of an algebraic number $\alpha$ by

$$
M(\alpha)=H(\alpha)^{[\mathbb{Q}(\alpha): \mathbb{Q}]} .
$$

Since $H$ is invariant under Galois conjugation over $\mathbb{Q}$, we obtain immediately

$$
M(\alpha)=\prod_{n=1}^{N} H\left(\alpha_{n}\right),
$$

2000 Mathematics Subject Classification. Primary 11R04, 11R09.

Key words and phrases. Weil height, Mahler measure, metric Mahler measure, Lehmer's problem. 
where $\alpha_{1}, \ldots, \alpha_{N}$ are the conjugates of $\alpha$ over $\mathbb{Q}$. Further, it is well-known that

$$
M(\alpha)=|A| \cdot \prod_{n=1}^{N} \max \left\{1,\left|\alpha_{n}\right|\right\},
$$

where $|\cdot|$ denotes the usual absolute value on $\mathbb{C}$. While the right hand side of (1.4) appears initially to depend upon a particular embedding of $\overline{\mathbb{Q}}$ into $\mathbb{C}$, any change of embedding simply permutes the images of the points $\left\{\alpha_{n}\right\}$ so that (1.4) remains unchanged.

It follows, again from Kronecker's Theorem, that $M(\alpha)=1$ if and only if $\alpha$ is zero or a root of unity. As part of an algorithm for computing large primes, D.H. Lehmer [5] asked whether there exists a constant $c>1$ such that $M(\alpha) \geq c$ in all other cases. The smallest known Mahler measure greater than 1 occurs at a root of

$$
\ell(x)=x^{10}+x^{9}-x^{7}-x^{6}-x^{5}-x^{4}-x^{3}+x+1
$$

which has Mahler measure 1.17.... Although an affirmative answer to Lehmer's problem has been given in many special cases, the general case remains open. The best known universal lower bound on $M(\alpha)$ is due to Dobrowolski [1, who proved that

$$
\log M(\alpha) \gg\left(\frac{\log \log \operatorname{deg} \alpha}{\log \operatorname{deg} \alpha}\right)^{3}
$$

whenever $\alpha$ is not a root of unity.

Recently, Dubickas and Smyth [2] defined the metric Mahler measure of an algebraic number $\alpha$ by

$$
M_{1}(\alpha)=\inf \left\{\prod_{n=1}^{N} M\left(\alpha_{n}\right): N \in \mathbb{N}, \alpha_{n} \in \overline{\mathbb{Q}}^{\times}, \alpha=\prod_{n=1}^{N} \alpha_{n}\right\} .
$$

Here, the infimum is taken over all ways to represent $\alpha$ as a product of elements in $\overline{\mathbb{Q}}^{\times}$. It is easily verified that

$$
M_{1}(\alpha \beta) \leq M_{1}(\alpha) M_{1}(\beta)
$$

for all $\alpha, \beta \in \overline{\mathbb{Q}}^{\times}$, and further, $M_{1}$ is well-defined on the quotient group $\mathcal{G}=$ $\overline{\mathbb{Q}}^{\times} / \operatorname{Tor}\left(\overline{\mathbb{Q}}^{\times}\right)$. This implies that the map $(\alpha, \beta) \mapsto \log M_{1}\left(\alpha \beta^{-1}\right)$ defines a metric on $\mathcal{G}$ which induces the discrete topology if and only if there is an affirmative answer to Lehmer's problem.

Also in 2, Dubickas and Smyth conjecture that the infimum in the definition of $M_{1}$ is always achieved. We verify this conjecture as well as explicitly determine a set in which the infimum must occur.

If $K$ is any number field let

$$
\operatorname{Rad}(K)=\left\{\alpha \in \overline{\mathbb{Q}}^{\times}: \alpha^{r} \in K \text { for some } r \in \mathbb{N}\right\},
$$

the set of all roots of points in $K$. Also, for the remainder of this paper, we write $K_{\alpha}$ for the Galois closure of $\mathbb{Q}(\alpha)$ over $\mathbb{Q}$.

Theorem 1.1. If $\alpha$ is a non-zero algebraic number then there exist $\alpha_{1}, \ldots, \alpha_{N} \in$ $\operatorname{Rad}\left(K_{\alpha}\right)$ such that $\alpha=\alpha_{1} \cdots \alpha_{N}$ and $M_{1}(\alpha)=M\left(\alpha_{1}\right) \cdots M\left(\alpha_{N}\right)$. 
Motivated by the work of Dubickas and Smyth, Fili and the author [4] defined a non-Archimedean version of $M_{1}$ by replacing the product in (1.6) by a maximum. That is, define the ultrametric Mahler measure by

$$
M_{\infty}(\alpha)=\inf \left\{\max _{1 \leq n \leq N} M\left(\alpha_{n}\right): N \in \mathbb{N}, \alpha_{n} \in \overline{\mathbb{Q}}^{\times}, \alpha=\prod_{n=1}^{N} \alpha_{n}\right\} .
$$

It easily verified that $M_{\infty}$ satisfies the strong triangle inequality

$$
M_{\infty}(\alpha \beta) \leq \max \left\{M_{\infty}(\alpha), M_{\infty}(\beta)\right\}
$$

for all non-zero algebraic numbers $\alpha$ and $\beta$. It is further shown in [4] that $M_{\infty}$ is well-defined on the quotient group $\mathcal{G}$. We can now establish the obvious analog of Theorem 1.1 for $M_{\infty}$.

Theorem 1.2. If $\alpha$ is a non-zero algebraic number then there exist $\alpha_{1}, \ldots, \alpha_{N} \in$ $\operatorname{Rad}\left(K_{\alpha}\right)$ such that $\alpha=\alpha_{1} \cdots \alpha_{N}$ and $M_{\infty}(\alpha)=\max \left\{M\left(\alpha_{1}\right), \ldots, M\left(\alpha_{N}\right)\right\}$.

The remainder of this paper is organized in the following way. Section 2 contains the core of our argument in which we show that computing $M_{1}(\alpha)$ and $M_{\infty}(\alpha)$ requires only the use of elements in $\operatorname{Rad}\left(K_{\alpha}\right)$. In section 3, we finish the proofs of Theorems 1.1 and 1.2 by showing, essentially, that there are only finitely many values for the Mahler measure in $\operatorname{Rad}\left(K_{\alpha}\right)$. Finally, section 4 contains some applications of these results, giving the location of the algebraic numbers $M_{1}(\alpha)$ and $M_{\infty}(\alpha)$.

\section{REDUCING TO SIMPLER REPRESENTATIONS}

The main idea in both proofs involves a method for replacing an arbitrary representation of $\alpha$ by a potentially smaller representation containing only points in $\operatorname{Rad}\left(K_{\alpha}\right)$. This technique is summarized by the following result.

Theorem 2.1. If $\alpha, \alpha_{1}, \ldots, \alpha_{N}$ are non-zero algebraic numbers with $\alpha=\alpha_{1} \cdots \alpha_{N}$ then there exists a root of unity $\zeta$ and algebraic numbers $\beta_{1}, \ldots, \beta_{N}$ satifying

(i) $\alpha=\zeta \beta_{1} \cdots \beta_{N}$,

(ii) $\beta_{n} \in \operatorname{Rad}\left(K_{\alpha}\right)$ for all $n$,

(iii) $M\left(\beta_{n}\right) \leq M\left(\alpha_{n}\right)$ for all $n$.

It is worth noting that we are unaware of an example in which computing $M_{1}(\alpha)$ or $M_{\infty}(\alpha)$ requires the use of elements outside $K_{\alpha}$. Hence, it seems reasonable to believe that we can, in fact, choose the points $\beta_{n}$ to belong to $K_{\alpha}$. Unfortunately, our proof suggests no way to verify this.

The proof of Theorem 2.1 is based on the following lemma.

Lemma 2.2. Suppose that $K$ is Galois over $\mathbb{Q}$. If $\gamma$ is an algebraic number then

$$
[K(\gamma): K]=[\mathbb{Q}(\gamma): K \cap \mathbb{Q}(\gamma)] .
$$

Moreover, we have that

$$
\prod_{n=1}^{N} \gamma_{n} \in K \cap \mathbb{Q}(\gamma)
$$

where $\gamma_{1}, \ldots, \gamma_{N}$ are the conjugates of $\gamma$ over $K$. 
Proof. We see clearly that $K(\gamma)$ is the compositum of $K$ and $\mathbb{Q}(\gamma)$. Since $K$ is Galois over $\mathbb{Q}$, it follows (see 3 , p. 505, Prop. 19) that $[K(\gamma): K]=[\mathbb{Q}(\gamma)$ : $K \cap \mathbb{Q}(\gamma)$ ], verifying (2.1). We also observe that

$$
(K \cap \mathbb{Q}(\gamma))(\gamma) \subseteq(\mathbb{Q}(\gamma))(\gamma)=\mathbb{Q}(\gamma) \subseteq(K \cap \mathbb{Q}(\gamma))(\gamma)
$$

so we conclude from (2.1) that

$$
[K(\gamma): K]=[(K \cap \mathbb{Q}(\gamma))(\gamma): K \cap \mathbb{Q}(\gamma)] .
$$

Let $f$ be the monic minimal polynomial of $\gamma$ over $K \cap \mathbb{Q}(\gamma)$ so that $f$ has degree $D$ equal to both sides of (2.3). Now write

$$
f(x)=x^{D}+\cdots+a_{1} x+a_{0}
$$

and note that $f$ is, of course, a polynomial over $K$. In fact, $f$ is the monic minimal polynomial of $\gamma$ over $K$ because it vanishes at $\gamma$ and has degree $[K(\gamma): K]$. Since $\gamma_{1}, \ldots, \gamma_{N}$ are the conjugates of $\gamma$ over $K$ we conclude that

$$
\prod_{n=1}^{N} \gamma_{n}= \pm a_{0}
$$

which belongs to $K \cap \mathbb{Q}(\gamma)$.

It is worth observing that if $\mathbb{Q}(\gamma)$ is Galois over $\mathbb{Q}$, then Lemma 2.2 becomes trivial. Indeed, $\gamma_{1} \cdots \gamma_{N}$ certainly belongs to $K$ by definition. But also, if $\mathbb{Q}(\gamma)$ is Galois, then $\mathbb{Q}(\gamma)$ contains all conjugates of $\gamma$ over $\mathbb{Q}$. In particular, it contains $\gamma_{n}$ for all $n$, so it contains their product as well. Of course, the proof of Theorem 2.1 does not permit such a hypothesis, so we require the above lemma.

Additionally, we cannot omit the hypothesis that $K$ be Galois over $\mathbb{Q}$. For example, let $\gamma_{1}, \gamma_{2}$ and $\gamma_{3}$ be the roots of a third degree, irreducible polynomial over $\mathbb{Q}$ having Galois group $S_{3}$. This means that $\mathbb{Q}\left(\gamma_{1}\right) \cap \mathbb{Q}\left(\gamma_{2}\right)=\mathbb{Q}$. Further, we observe that $\gamma_{2}$ must have degree 2 over $\mathbb{Q}\left(\gamma_{1}\right)$ implying that its conjugates over this field are $\gamma_{2}$ and $\gamma_{3}$. But if $\gamma_{2} \cdot \gamma_{3} \in \mathbb{Q}\left(\gamma_{2}\right)$ then $\gamma_{1} \in \mathbb{Q}\left(\gamma_{2}\right)$, a contradiction.

Proof of Theorem [2.1. Suppose that $\alpha=\alpha_{1} \cdots \alpha_{N}$ and let $E$ be a Galois extension of $K_{\alpha}$ containing $\alpha_{n}$ for all $n$. Let $G=\operatorname{Gal}\left(E / K_{\alpha}\right), G_{n}=\operatorname{Gal}\left(E / K_{\alpha}\left(\alpha_{n}\right)\right)$ and $S_{n}$ a set of left coset representatives of $G_{n}$ in $G$. We have that

$$
\begin{aligned}
\alpha^{\left[E: K_{\alpha}\right]} & =\operatorname{Norm}_{E / K_{\alpha}}(\alpha) \\
& =\prod_{n=1}^{N} \operatorname{Norm}_{E / K_{\alpha}}\left(\alpha_{n}\right) \\
& =\prod_{n=1}^{N} \prod_{\sigma \in G} \sigma\left(\alpha_{n}\right) \\
& =\prod_{n=1}^{N} \prod_{\sigma \in S_{n}} \prod_{\tau \in G_{n}} \sigma\left(\tau\left(\alpha_{n}\right)\right) \\
& =\prod_{n=1}^{N} \prod_{\sigma \in S_{n}} \sigma\left(\alpha_{n}\right)^{\left|G_{n}\right|}
\end{aligned}
$$


so we conclude that

$$
\alpha^{\left[E: K_{\alpha}\right]}=\prod_{n=1}^{N}\left(\prod_{\sigma \in S_{n}} \sigma\left(\alpha_{n}\right)\right)^{\left[E: K_{\alpha}\left(\alpha_{n}\right)\right]} .
$$

For each $n$, we select an element $\beta_{n} \in \overline{\mathbb{Q}}$ such that

$$
\beta_{n}^{\left[K_{\alpha}\left(\alpha_{n}\right): K_{\alpha}\right]}=\prod_{\sigma \in S_{n}} \sigma\left(\alpha_{n}\right)
$$

so that, in view of (2.4), we obtain

$$
\alpha^{\left[E: K_{\alpha}\right]}=\prod_{n=1}^{N} \beta_{n}^{\left[E: K_{\alpha}\right]}
$$

This implies the existence of a root of unity $\zeta$ such that

$$
\alpha=\zeta \beta_{1} \cdots \beta_{N}
$$

Furthermore, the set $\left\{\sigma\left(\alpha_{n}\right): \sigma \in S_{n}\right\}$ is precisely the set of conjugates of $\alpha_{n}$ over $K_{\alpha}$ so that

$$
\prod_{\sigma \in S_{n}} \sigma\left(\alpha_{n}\right) \in K_{\alpha}
$$

It then follows from (2.5) that $\beta_{n} \in \operatorname{Rad}\left(K_{\alpha}\right)$ for each $n$ as well.

It remains to show that $M\left(\beta_{n}\right) \leq M\left(\alpha_{n}\right)$ for all $n$. To see this, we note that (2.5) yields immediately

$$
\operatorname{deg}\left(\beta_{n}\right) \leq\left[K_{\alpha}\left(\alpha_{n}\right): K_{\alpha}\right] \cdot \operatorname{deg}\left(\prod_{\sigma \in S_{n}} \sigma\left(\alpha_{n}\right)\right) .
$$

Once again, the elements $\sigma\left(\alpha_{n}\right)$ for $\sigma \in S_{n}$ are precisely the conjugates of $\alpha_{n}$ over $K_{\alpha}$. Hence, we may apply Lemma $2.2(2.1)$ to find that

$$
\prod_{\sigma \in S_{n}} \sigma\left(\alpha_{n}\right) \in K_{\alpha} \cap \mathbb{Q}\left(\alpha_{n}\right) .
$$

Combining this with (2.7), we obtain

$$
\operatorname{deg}\left(\beta_{n}\right) \leq\left[K_{\alpha}\left(\alpha_{n}\right): K_{\alpha}\right] \cdot\left[K_{\alpha} \cap \mathbb{Q}\left(\alpha_{n}\right): \mathbb{Q}\right]
$$

Then we find that

$$
\begin{aligned}
M\left(\beta_{n}\right) & \leq H\left(\beta_{n}\right)^{\left[K_{\alpha}\left(\alpha_{n}\right): K_{\alpha}\right] \cdot\left[K_{\alpha} \cap \mathbb{Q}\left(\alpha_{n}\right): \mathbb{Q}\right]} \\
& =H\left(\prod_{\sigma \in S_{n}} \sigma\left(\alpha_{n}\right)\right)^{\left[K_{\alpha} \cap \mathbb{Q}\left(\alpha_{n}\right): \mathbb{Q}\right]} \\
& \leq H\left(\alpha_{n}\right)^{\left[K_{\alpha}\left(\alpha_{n}\right): K_{\alpha}\right] \cdot\left[K_{\alpha} \cap \mathbb{Q}\left(\alpha_{n}\right): \mathbb{Q}\right]}
\end{aligned}
$$

where the last inequality follows since the Weil height is invariant under Galois conjugation and satisfies the triangle inequality. But also $K_{\alpha}\left(\alpha_{n}\right)$ is the compositum of $K_{\alpha}$ and $\mathbb{Q}\left(\alpha_{n}\right)$ so that $\left[K_{\alpha}\left(\alpha_{n}\right): K_{\alpha}\right]=\left[\mathbb{Q}\left(\alpha_{n}\right): K_{\alpha} \cap \mathbb{Q}\left(\alpha_{n}\right)\right]$ by (2.1) in Lemma 2.2. This yields

$$
M\left(\beta_{n}\right) \leq H\left(\alpha_{n}\right)^{\left[\mathbb{Q}\left(\alpha_{n}\right): K_{\alpha} \cap \mathbb{Q}\left(\alpha_{n}\right)\right] \cdot\left[K_{\alpha} \cap \mathbb{Q}\left(\alpha_{n}\right): \mathbb{Q}\right]}=M\left(\alpha_{n}\right)
$$

which completes the proof. 


\section{Proofs of Theorems 1.1 and 1.2}

In view of Theorem 2.1, it is enough, in the definitions of $M_{1}$ and $M_{\infty}$, to consider only representations $\alpha=\alpha_{1} \cdots \alpha_{N}$ having $\alpha_{n} \in \operatorname{Rad}\left(K_{\alpha}\right)$ for all $n$. Any representation that fails to have this property may simply be replaced a smaller represenation that does. The remainder of our proofs of both Theorem 1.1 and 1.2 require us to show that such representations yield only finitely many different values for

$$
\max _{1 \leq n \leq N} M\left(\alpha_{n}\right) \text { and } \prod_{n=1}^{N} M\left(\alpha_{n}\right) .
$$

The following lemma provides the starting point for this argument.

Lemma 3.1. Let $K$ be a Galois extension of $\mathbb{Q}$. If $\gamma \in \operatorname{Rad}(K)$ then there exists a root of unity $\zeta$ and $L, S \in \mathbb{N}$ such that $\zeta \gamma^{L} \in K$ and

$$
M(\gamma)=M\left(\zeta \gamma^{L}\right)^{S} .
$$

In particular, the set

$$
\{M(\gamma): \gamma \in \operatorname{Rad}(K), M(\gamma) \leq B\}
$$

is finite for every $B \geq 1$.

Proof. Suppose that $\gamma^{r} \in K$, so that each conjugate of $\gamma$ over $K$ must be a root of $x^{r}-\gamma^{r} \in K[x]$. Therefore, we may assume that $\gamma$ has conjugates

$$
\zeta_{1} \gamma, \ldots, \zeta_{L} \gamma
$$

over $K$ for some roots of unity $\zeta_{1}, \ldots, \zeta_{L}$. By Lemma 2.2 we conclude that

$$
\zeta_{1} \cdots \zeta_{L} \gamma^{L}=\zeta_{1} \gamma \cdots \zeta_{L} \gamma \in K \cap \mathbb{Q}(\gamma) .
$$

Since $K$ is Galois, Lemma 2.2 also implies that $L=[K(\gamma): K]=[\mathbb{Q}(\gamma): K \cap \mathbb{Q}(\gamma)]$. Hence, we find that

$$
\begin{aligned}
M(\gamma) & =H(\gamma)^{[\mathbb{Q}(\gamma): \mathbb{Q}]} \\
& =H(\gamma)^{[\mathbb{Q}(\gamma): K \cap \mathbb{Q}(\gamma)] \cdot[K \cap \mathbb{Q}(\gamma): \mathbb{Q}]} \\
& =H(\gamma)^{L \cdot[K \cap \mathbb{Q}(\gamma): \mathbb{Q}]} .
\end{aligned}
$$

Since $L$ is a positive integer and $\zeta_{1} \cdots \zeta_{L}$ is a root of unity, we conclude from (1.1) and (1.2) that

$$
M(\gamma)=H\left(\zeta_{1} \cdots \zeta_{L} \gamma^{L}\right)^{[K \cap \mathbb{Q}(\gamma): \mathbb{Q}]} .
$$

By (3.1) we know that there exists a positive integer $S$ such that

$$
[K \cap \mathbb{Q}(\gamma): \mathbb{Q}]=S \cdot\left[\mathbb{Q}\left(\zeta_{1} \cdots \zeta_{L} \gamma^{L}\right): \mathbb{Q}\right]
$$

and so (3.2) yields

$$
M(\gamma)=H\left(\zeta_{1} \cdots \zeta_{L} \gamma^{L}\right)^{S \cdot\left[\mathbb{Q}\left(\zeta_{1} \cdots \zeta_{L} \gamma^{L}\right): \mathbb{Q}\right]}=M\left(\zeta_{1} \cdots \zeta_{L} \gamma^{L}\right)^{S} .
$$

Taking $\zeta=\zeta_{1} \cdots \zeta_{L}$ we have that $\zeta \gamma^{L} \in K$ by (3.1) and $M(\gamma)=M\left(\zeta \gamma^{L}\right)^{S}$ which establishes the first statement of the lemma.

Further, we note that (3.2) implies that

$$
M(\gamma)=H\left(\left(\zeta \gamma^{L}\right)^{[K \cap \mathbb{Q}(\gamma): \mathbb{Q}]}\right) .
$$


But $\left(\zeta \gamma^{L}\right)^{[K \cap \mathbb{Q}(\gamma): \mathbb{Q}]} \in K$ implying that

$$
\{M(\gamma): \gamma \in \operatorname{Rad}(K), M(\gamma) \leq B\} \subseteq\left\{H(\alpha): \alpha \in K^{\times}, H(\alpha) \leq B\right\} .
$$

It follows from Northcott's Theorem [6] that the right hand side of (3.3) is finite, completing the proof.

The proof of Theorem 1.2 is somewhat simpler than that of Theorem 1.1 so we include it here first.

Proof of Theorem 1.2. There exists $\varepsilon>0$ such that if $\alpha=\alpha_{1} \cdots \alpha_{N}$ with $\alpha_{n} \in$ $\operatorname{Rad}\left(K_{\alpha}\right)$ and

$$
M_{\infty}(\alpha) \leq \max \left\{M\left(\alpha_{1}\right), \ldots, M\left(\alpha_{N}\right)\right\} \leq M_{\infty}(\alpha)+\varepsilon
$$

then $M_{\infty}(\alpha)=\max \left\{M\left(\alpha_{1}\right), \ldots, M\left(\alpha_{N}\right)\right\}$. Otherwise, we get a sequence $\left\{x_{m}\right\} \subseteq$ $\operatorname{Rad}\left(K_{\alpha}\right)$ such that $\left\{M\left(x_{m}\right)\right\}$ is strictly decreasing, contradicting Lemma 3.1

By definition, there exists a representation $\alpha=\gamma_{1} \cdots \gamma_{N}$ with

$$
M_{\infty}(\alpha) \leq \max \left\{M\left(\gamma_{1}\right), \ldots, M\left(\gamma_{N}\right)\right\} \leq M_{\infty}(\alpha)+\varepsilon .
$$

By Theorem 2.1, there exists a representation $\alpha=\zeta \alpha_{1} \cdots \alpha_{N}$ such that $\zeta$ is a root of unity, $\alpha_{n} \in \operatorname{Rad}\left(K_{\alpha}\right)$ and and $M\left(\alpha_{n}\right) \leq M\left(\gamma_{n}\right)$ for all $n$. This yields

$$
M_{\infty}(\alpha) \leq \max \left\{M\left(\alpha_{1}\right), \ldots, M\left(\alpha_{N}\right)\right\} \leq M_{\infty}(\alpha)+\varepsilon
$$

so that $M_{\infty}(\alpha)=\max \left\{M\left(\alpha_{1}\right), \ldots, M\left(\alpha_{N}\right)\right\}$ by our earlier remarks.

We note that the above proof is not sufficient to establish Theorem 1.1. Indeed, Lemma 3.1 does not prevent the product $M\left(\alpha_{1}\right) \cdots M\left(\alpha_{N}\right)$ from having infinitely many values between $M_{1}(\alpha)$ and $M_{1}(\alpha)+\varepsilon$ unless we can bound $N$ uniformly from above by a function of $\alpha$.

In order to do this, we introduce an additional definition. We say that a representation $\alpha=\alpha_{1} \cdots \alpha_{N}$ is $B$-restricted if the following three conditions hold.

(i) $M\left(\alpha_{1}\right) \cdots M\left(\alpha_{N}\right) \leq B$

(ii) $\alpha_{n} \in \operatorname{Rad}\left(K_{\alpha}\right)$ for all $n$

(iii) At most one element $\alpha_{n}$ is a root of unity.

We write $R_{B}(\alpha)$ to denote the set of all $N$-tuples, for all $N \in \mathbb{N}$, of non-zero algebraic numbers that form $B$-restricted representations of $\alpha$. Further, set

$$
q(\alpha)=\inf \left\{H(x): x \in K_{\alpha}^{\times} \backslash \operatorname{Tor}\left(\overline{\mathbb{Q}}^{\times}\right)\right\}
$$

and note that, by Northcott's Theorem [6], this quantity is always strictly greater than 1. Using these definitions, we obtain the result we need to finish the proof of Theorem 1.1.

Lemma 3.2. Let $\alpha$ be a non-zero algebraic number and $B \geq 1$. If $\alpha=\alpha_{1} \cdots \alpha_{N}$ is an $B$-restricted representation of $\alpha$ then

$$
N \leq 1+\frac{\log B}{\log q(\alpha)} .
$$

Moreover, the set

$$
\left\{\prod_{n=1}^{N} M\left(\alpha_{n}\right): N \in \mathbb{N},\left(\alpha_{1}, \ldots, \alpha_{N}\right) \in R_{B}(\alpha)\right\}
$$

is finite. 
Proof. Suppose that $\alpha=\alpha_{1} \cdots \alpha_{N}$ is an $B$-restricted representation. By assumption, at least $N-1$ of the terms $\alpha_{n}$ in our representation are not roots of unity. Assume $\alpha_{n}$ is one such element. Lemma 3.1 implies that there exists a point $\gamma_{n} \in K_{\alpha}$ such that

$$
M\left(\alpha_{n}\right)=H\left(\gamma_{n}\right) .
$$

Since $\alpha_{n}$ is not a root of unity, neither side of (3.4) equals 1 , so that $\gamma_{n}$ is not a root of unity either. Therefore, we find that $M\left(\alpha_{n}\right) \geq q(\alpha)$ for $N-1$ of the terms belonging to $\left\{\alpha_{1}, \ldots, \alpha_{N}\right\}$. This yields

$$
B \geq M\left(\alpha_{1}\right) \cdots M\left(\alpha_{N}\right) \geq q(\alpha)^{N-1} .
$$

We know that $q(\alpha)>1$ so that we may divide by $\log q(\alpha)$ to obtain

$$
N \leq 1+\frac{\log B}{\log q(\alpha)}
$$

verifying the first statement of the lemma. We now find that

$$
\begin{aligned}
\left\{\prod_{n=1}^{N} M\left(\alpha_{n}\right):\right. & \left.N \in \mathbb{N},\left(\alpha_{1}, \ldots, \alpha_{N}\right) \in R_{B}(\alpha)\right\} \\
& =\left\{\prod_{n=1}^{N} M\left(\alpha_{n}\right):\left(\alpha_{1}, \ldots, \alpha_{N}\right) \in R_{B}(\alpha), N \leq 1+\frac{\log B}{\log q(\alpha)}\right\} \\
& \subseteq\left\{\prod_{n=1}^{N} M\left(\alpha_{n}\right): N \leq 1+\frac{\log B}{\log q(\alpha)}, M\left(\alpha_{n}\right) \leq B, \alpha_{n} \in \operatorname{Rad}\left(K_{\alpha}\right)\right\}
\end{aligned}
$$

which is finite by Lemma 3.1 .

Proof of Theorem 1.1. By Lemma 3.2, we may select $B>M_{1}(\alpha)$ such that

$$
\left(M_{1}(\alpha), B\right) \bigcap\left\{\prod_{n=1}^{N} M\left(\alpha_{n}\right): N \in \mathbb{N},\left(\alpha_{1}, \ldots, \alpha_{N}\right) \in R_{M_{1}(\alpha)+1}(\alpha)\right\}=\emptyset .
$$

Of course, we may choose $B \leq M_{1}(\alpha)+1$ which gives

$$
\begin{aligned}
\left\{\prod_{n=1}^{N} M\left(\alpha_{n}\right):\right. & \left.N \in \mathbb{N},\left(\alpha_{1}, \ldots, \alpha_{N}\right) \in R_{B}(\alpha)\right\} \\
& \subseteq\left\{\prod_{n=1}^{N} M\left(\alpha_{n}\right): N \in \mathbb{N},\left(\alpha_{1}, \ldots, \alpha_{N}\right) \in R_{M_{1}(\alpha)+1}(\alpha)\right\},
\end{aligned}
$$

and therefore,

$$
\left(M_{1}(\alpha), B\right) \bigcap\left\{\prod_{n=1}^{N} M\left(\alpha_{n}\right): N \in \mathbb{N},\left(\alpha_{1}, \ldots, \alpha_{N}\right) \in R_{B}(\alpha)\right\}=\emptyset .
$$

By definition of $M_{1}$, there exists a representation $\alpha=\gamma_{1} \cdots \gamma_{L}$ such that

$$
M_{1}(\alpha) \leq M\left(\gamma_{1}\right) \cdots M\left(\gamma_{L}\right)<B .
$$

Theorem 2.1 implies that there exists a representation $\alpha=\zeta \beta_{1} \cdots \beta_{L}$ with $\zeta$ a root of unity, each element $\beta_{\ell}$ belonging to $\operatorname{Rad}\left(K_{\alpha}\right)$ and $M\left(\beta_{\ell}\right) \leq M\left(\gamma_{\ell}\right)$ for all $\ell$. This yields

$$
M_{1}(\alpha) \leq M(\zeta) M\left(\beta_{1}\right) \cdots M\left(\beta_{L}\right)<B .
$$


By combining all roots of unity in the representation into a single element, we obtain a new representation $\alpha=\alpha_{1} \cdots \alpha_{N}$ having $\alpha_{n} \in \operatorname{Rad}\left(K_{\alpha}\right)$, at most one root of unity, and

$$
M\left(\alpha_{1}\right) \cdots M\left(\alpha_{N}\right)=M\left(\beta_{1}\right) \cdots M\left(\beta_{L}\right) .
$$

Therefore, we see that

$$
M_{1}(\alpha) \leq M\left(\alpha_{1}\right) \cdots M\left(\alpha_{N}\right)<B
$$

which implies, in particular, that $\left(\alpha_{1}, \ldots, \alpha_{N}\right) \in R_{B}(\alpha)$. Then by (3.6) we get that

$$
M\left(\alpha_{1}\right) \cdots M\left(\alpha_{N}\right) \notin\left(M_{1}(\alpha), B\right) .
$$

Finally, combining (3.7) and (3.8) we obtain $M_{1}(\alpha)=M\left(\alpha_{1}\right) \cdots M\left(\alpha_{N}\right)$.

\section{The Location of $M_{1}(\alpha)$ And $M_{\infty}(\alpha)$}

We now apply Theorems 1.1 and 1.2 in order to show that $M_{1}(\alpha)$ and $M_{\infty}(\alpha)$ belong to $K_{\alpha}$. We begin with $M_{\infty}$ in which case we are able to prove a slightly stronger result.

Theorem 4.1. If $\alpha$ is an algebraic number then there exists $\beta \in K_{\alpha}$ such that $M_{\infty}(\alpha)=M(\beta)$. In particular, $M_{\infty}(\alpha) \in K_{\alpha}$.

Proof. By Theorem 1.2 there exist $\alpha_{1}, \ldots, \alpha_{N} \in \operatorname{Rad}\left(K_{\alpha}\right)$ such that $\alpha=\alpha_{1} \cdots \alpha_{N}$ and

$$
M_{\infty}(\alpha)=\max \left\{M\left(\alpha_{1}\right), \ldots, M\left(\alpha_{N}\right)\right\} .
$$

For each $n$, Lemma 3.1 implies that there exists a root of unity $\zeta_{n}$ and $L_{n}, S_{n} \in \mathbb{N}$ such that

$$
M\left(\alpha_{n}\right)=M\left(\zeta_{n} \alpha_{n}^{L_{n}}\right)^{S_{n}}
$$

and $\zeta_{n} \alpha_{n}^{L_{n}} \in K_{\alpha}$. For simplicity, we write

$$
L=\prod_{n=1}^{N} L_{n} \quad \text { and } \quad J_{n}=\prod_{k \neq n} L_{k}
$$

so that $L=L_{n} J_{n}$ for all $n$. Then we obtain immediately

$$
\alpha^{L}=\prod_{n=1}^{N} \alpha_{n}^{L_{n} J_{n}}
$$

so there exists a root of unity $\zeta$ such that

$$
\zeta \alpha^{L}=\prod_{n=1}^{N}\left(\zeta_{n} \alpha_{n}^{L_{n}}\right)^{J_{n}} .
$$

By Theorem 1.3 of 4 , we obtain that

$$
\begin{aligned}
M_{\infty}(\alpha) & =M_{\infty}\left(\zeta \alpha^{L}\right) \\
& \leq \max _{1 \leq n \leq N}\left\{M\left(\zeta_{n} \alpha_{n}^{L_{n}}\right)\right\} \\
& \leq \max _{1 \leq n \leq N}\left\{M\left(\zeta_{n} \alpha_{n}^{L_{n}}\right)^{S_{n}}\right\} \\
& =\max _{1 \leq n \leq N}\left\{M\left(\alpha_{n}\right)\right\} \\
& =M_{\infty}(\alpha) .
\end{aligned}
$$


Therefore, we have that $M_{\infty}(\alpha)=\max _{1 \leq n \leq N}\left\{M\left(\zeta_{n} \alpha_{n}^{L_{n}}\right)\right\}$. As we have noted, each element $\zeta_{n} \alpha_{n}^{L_{n}}$ belongs to $K_{\alpha}$ completing the proof of the first statement.

Now we have that $M_{\infty}(\alpha)=M(\beta)$ for some $\beta \in K_{\alpha}$. Since $K_{\alpha}$ is Galois, it must contain all conjugates of $\beta$ over $\mathbb{Q}$, and therefore, it contains the product of all roots outside the unit circle. This product is a real number so $K_{\alpha}$ must contain its absolute value. Hence we get that $M_{\infty}(\alpha) \in K_{\alpha}$.

In the case of $M_{1}$, we cannot establish a result as strong as Theorem 4.1, but we can prove an analog of its second statement.

Theorem 4.2. If $\alpha$ is an algebraic number then $M_{1}(\alpha) \in K_{\alpha}$.

Proof. By Theorem 1.1 we know that there exist $\alpha_{1}, \ldots, \alpha_{N} \in \operatorname{Rad}\left(K_{\alpha}\right)$ such that $\alpha=\alpha_{1} \cdots \alpha_{N}$ and

$$
M_{1}(\alpha)=M\left(\alpha_{1}\right) \cdots M\left(\alpha_{N}\right) .
$$

According to Lemma 3.1, for each $n$ there exists an algebraic number $\gamma_{n} \in K_{\alpha}$ and a positive integer $S_{n}$ such that $M\left(\alpha_{n}\right)=M\left(\gamma_{n}\right)^{S_{n}}$. Each conjugate of $\gamma$ over $\mathbb{Q}$ must belong to the Galois extension $K_{\alpha}$, which implies that $M\left(\gamma_{n}\right) \in K_{\alpha}$ for all $n$. It follows that $M_{1}(\alpha) \in K_{\alpha}$.

\section{REFERENCES}

[1] E. Dobrowolski, On a question of Lehmer and the number of irreducible factors of a polynomial, Acta Arith. 34 (1979), no. 4, 391-401.

[2] A. Dubickas and C.J. Smyth, On the metric Mahler measure, J. Number Theory 86 (2001), 368-387.

[3] D.S. Dummit and R.M. Foote, Abstract Algebra, Prentice Hall, Englewood Cliffs, NJ (1991).

[4] P. Fili and C.L. Samuels, On the non-Archimedean metric Mahler measure, preprint (2008).

[5] D.H. Lehmer, Factorization of certain cyclotomic functions, Ann. of Math. 34 (1933), 461-479.

[6] D.G. Northcott, An inequality on the theory of arithmetic on algebraic varieties, Proc. Cambridge Philos. Soc., 45 (1949), 502-509.

Max-Planck-Institut für Mathematik, Vivatsqasse 7, 53111 Bonn, Germany

E-mail address: csamuels@mpim-bonn.mpg.de 